\title{
and antihyperglycemia effects of resvertrol and its derivative on alloxan diabetic rabbits
}

\author{
Essam F.Al-Jumaily ${ }^{1}$ Redha I. Al-Bayati ${ }^{2}$ and Zuhair A. Shafiq ${ }^{1}$ \\ ${ }^{1}$ Biotechnology Dept. Genetic Engineering and Biotechnology Institute for postgraduate \\ studies-Baghdad University-Baghdad, Iraq \\ ${ }^{2}$ Chemistry Dept. Science College, Al-Msairitey University, Baghdad, Iraq
}

\begin{abstract}
:
Background and objective: Resveratrol is a non flavonoid polyphenol (Vitis vinifera) posses three phenolic hydroxyl group and shown to its biological effects. The derivations of resveratrol associated with the available oral hypoglycermic agents for treatment of diabetes mellutes type 2 ..

Materials and methods: The study has also employed an in vivo evaluation of resveratrol and its derivative in female rabbits at concentrations $(1 \mathrm{mg} / \mathrm{kg}$ ) given orally for 42 days after inducing diabetes mellitus type 2 by alloxan $(100 \mathrm{mg} / 1 \mathrm{~kg}$ body weight). The serum was isolated from heart blood for the biochemical tests, including Glucose, Total protein Albumin and Insulin. At day $\mathbf{4 2}$ the animal was killed and the liver and pancreas were kept in $\mathbf{1 0 \%}$ formalin for preparation of histopathological sections.

Results: Statistical analysis showed a significant decrease in Glucose, total protein , Creatinine of serum blood levels on treated rabbits $p<0.05$, However resveratrol and its derivative treated rabbits showed statistically significant increase in Insulin blood serum levels $p<0.05$. Examination of the liver tissue confirmed potential histopathological effects for resveratrol, while derivative has normal appearance in a dose dependent manner.

Examination of the pancreas tissue confirmed potential histopathological effects for resveratrol, and its derivatives has normal appearance in a dose dependent manner. The derivatives compound showed a significant differences than the other compounds extract regarding the serum activity of Insulin and hisopathological changes especially in pancreas.
\end{abstract}

Keywords-Reveratrol (non flavonoid polyphenol); hypoglycermic; Vitis vinifera ; hisopathological; glbcimaide

\section{INTRODUCTION}

Resveratrol (3,5,4 - trihydroxy stilbene), is a non Flavonoid polyphenol and it has three phenolic hydroxyl groups and shown to have its biological effects [1]. Phenolic and polyphenolic compounds, possess an aromatic ring bearing one or more hydroxyl substituents [2]. These compounds are phytoalexins and have antidiabeteic properties [3]. Resveratrol may offer benefits in preventing or managing conditions associated with high blood sugar [4]. The derivatives of resveratrol associated with the available oral hypoglycemic agents for the treatment of diabetes mellitus Epsilon-vinifera a resveratrol dimer, ,Piceatanol an active metabolic of resveratrol found in red wine, Piceid a resveratrol glucoside, Trans - diptoindonesin B a resveratrol trimer [5].

These plants produce trans-resveratrol to protect themselves after exposure to ultraviolet radiation, ozone or certain biologic agents. It functions as a ribonucleotide reductase inhibitor [6].The effects of resveratrol are currently a topic of numerous animal and human studies. Its effects on the lifespan of many model organisms remain controversial with uncertain effects in fruit flies, nematode worms and short-lived fish [7]. In the only positive human trial, extremely high doses $(3-5 \mathrm{~g})$ of resveratrol, in a proprietary formulation designed to enhance its bioavailability, significantly lowered blood sugar. Despite mainstream press alleging resveratrol's anti-aging effects, there is no accepted data to form a scientific basis for the application of these claims to mammals [8].

\section{MATERIALS AND METHODS}

\subsection{Plant materials}

Local black grapes cultivated Iraqi were collected from the local market and classified as Vitis vinifera by the herbarium of the Biology Department, College of Science, Baghdad University . The grape skin extract was prepared; all steps were done away from direct light and extensive stress that led to oxidation of the plant 
extract. About 500 grams of fresh skin grapes was shaken with 2.5 litters' $99.9 \%$ ethyl acetate in cool dark place for 72 hours. The extract was filtered and the filtrate was dried at $30-40^{\circ} \mathrm{C}$ by a rotary evaporator to get $1 / 10$ (one tenth) its original volume to be stored at $-20^{\circ} \mathrm{C}$ till the followings steps. The following steps were followed for the isolation of resveratrol:

\subsection{Preparation of Resveratrol Derivative}

5-(4-(4-(2-(benzo [d] thiazol-2-yl) hydrazinyl) butanoyloxy)styryl)-1,3-phenylene bis(4-(2-(benzo[d]thiazol-2yl) hydrazinyl)butanoate)

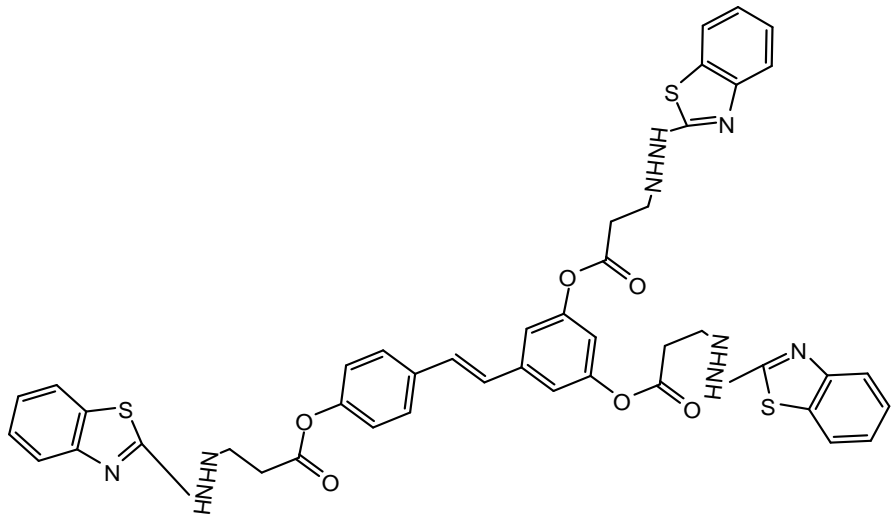

$1.6 \mathrm{gm}$ ( 0.003 mole) of compound (2) was dissolved in $50 \mathrm{ml}$ of absolute ethanol then $1.58 \mathrm{~g} 0.0096$ moles of 2 Mercptobenzothiazole then reflux for $8 \mathrm{~h}$. The solvent was removed and the precipitates was filtrated and dried

\subsection{Induction of Diabetes}

[9].

5,6-dioxyuracil,was used which marketing name (alloxan) and whose diabetogenic property is established with rabbits. The alloxan injection depends on the weight of the animal: $70 \mathrm{mg}$ of alloxan dissolved in $3 \mathrm{ml}$ of distilled water for $1 \mathrm{~kg}$ of rabbit weight. The alloxan solution is injected through the marginal vein of the ear after fasted for $24 \mathrm{~h}$ before injection. This single dose of alloxan produced type 1 diabetes having fasting blood sugar level of $155 \pm 10.71 \mathrm{mg} / \mathrm{dl}$ after10 days of injection of alloxan and this diabetic state was maintained throughout the 1 duration of the experiment .Therefore, the health status of rabbits was closely monitored until the hyperglycemia appeared and was detected weekly by determination of blood sugar quantity .

\subsection{Purified Resveratrol and its Derivative Dilutions}

Pure resveratrol $100 \mathrm{mg}$ was dissolved in $100 \mathrm{ml}$ PBS and $0.1 \mathrm{ml}$ DMSO as organic solvent for dissolving the substance. The stock was kept in a dark container at $-20^{\circ} \mathrm{C}$ after sterilization with $0.22 \mu \mathrm{m}$ Millipore filter.

Resveratrol derivative $50 \mathrm{mg}$ were dissolved in $0.05 \mathrm{ml}$ DMSO and complete the volume to $50 \mathrm{ml}$ with PBS. Then it was sterilized and kept in a dark container at $-20^{\circ} \mathrm{C}$.

\subsection{Experimental Design:}

Fifteen adult female rabbits were randomly divided into four groups and treated daily as follows for six weeks: The rabbits in first group received regular standard diet, tap water and served as control. After feeding them for about 1 week, their body weights and fasting blood sugar levels were taken. Other parameters which included levels were also taken and recorded.

\section{Group 2: Diabetic rabbits without treatment}

At the expiration of 1 week, alloxan was subject into the control group and they formed group (2) rabbits. The rabbits were confirmed diabetic after estimation of their fasting blood sugar level, 2 weeks after injection of alloxan. A rabbit was considered to be diabetic if it had a fasting blood sugar where level > 115 $\mathrm{mg} / \mathrm{dl}$. Other parameters were also taken and recorded.

\section{Group 3: Diabetic rabbits after treatment with glbcimaide}

At the expiration of 2 weeks of induction of diabetes into the rabbits of group (2), they were force fed with glbcimaide $0.05 \mathrm{mg}$ orally for a period of 6 weeks and, thus they formed the rabbits of group 3 . At the end of 4 weeks their fasting blood glucose levels was estimated and recorded. Other parameters were also taken and recorded.

\section{Group 4: Diabetic rabbits after treatment with Resveratrol}

At the expiration of 2 weeks of induction of diabetes into the rabbits of group (2), they were force fed with resveratrol $1 \mathrm{mg} / \mathrm{ml}$ orally for a period of 6 weeks and, thus they formed the rabbits of group 4 . At the end of 4 weeks their fasting blood glucose levels were estimated and recorded. Other parameters were also taken and recorded.

\section{Group 5: Diabetic rabbits after treatment with Resveratrol derivative}


At the expiration of 2 weeks of induction of diabetes into the rabbits of group (2), they were force fed with resveratrol derivative $1 \mathrm{mg} / \mathrm{ml}$ orally for a period of 6 weeks and they thus formed the rabbits of group (3). At the end of 4 weeks their fasting blood glucose levels was estimated and recorded. Other parameters were also taken and recorded.

\subsection{Biochemical Estimation:}

The fasting blood glucose levels was estimated according to (Young et al., 2000)[10] ; Total protein (Young et al., 2000 )[10]; serum triglyceride (Young et al.,2000)[10]; serum creatinine ( Young et al. 2000) [10] ; serum insulin ( Young et al.,2000)[10].

\subsection{Histopathological studies:}

Tissue samples from liver \& pancreas after delivery were prepared for histopathological studies according to the methods of Junqueira et al.[11].

\subsection{Statistical Analysis}

Data were analyzed by using the SPSS package programmed. Multiple range test was used to detect the significant differences [12].The method which was used to measure the significances at level 0.05 or 0.01 was taken from [13].

\section{RESULTS}

Table (1) shows the diabetic rabbits treated with the resveratrol and its derivative decreased significantly lower $(\mathrm{p}<0.05)$ in comparison with the control antidiabetic group after 3,4 and 6 weeks of the injection of the alloxan.For the rabbits in the groups treated with resveratrol and its derivatives, the food and water intake was decreased and the volume of urine decreased in comparison with the rabbits in the control antidiabetic groups. It can therefore be concluded that the preliminary study shows that the resveratrol and its derivative decreases the serum glucose level in diabetic rabbits. The resveratrol derivative shows significant value after one week of treatment $109.0 \mathrm{mg} / \mathrm{dL} \pm 2.5$ in comparison with resveratrol had normal values after 6 weeks.

Table (1): The effect of resveratrol and its derivative on serum glucose concentration $(\mathrm{mg} / \mathrm{dL})$ of rabbits' groups with weeks.

\begin{tabular}{|l|l|l|l|l|l|l|l|l|}
\hline \multirow{2}{*}{ Groups } & \multicolumn{6}{l}{ Treatment periods } \\
\cline { 2 - 9 } & WK0 & PT & Wk1 & Wk2 & Wk3 & Wk4 & Wk5 & Wk6 \\
& & & & & & & & \\
\hline C & $98 \pm$ & $98 \pm$ & $92.0 \pm 0$. & $96 \pm$ & $102 \pm$ & $101 \pm$ & $100 \pm$ & $99 \pm$ \\
& $2^{\mathrm{a}}$ & $2^{\mathrm{e}}$ & $5^{\mathrm{e}}$ & $2^{\mathrm{e}}$ & $1^{\mathrm{e}}$ & $0.5^{\mathrm{d}}$ & $0.5^{\mathrm{d}}$ & $3^{\mathrm{c}}$ \\
& $\mathrm{A}$ & $\mathrm{A}$ & $\mathrm{A}$ & $\mathrm{A}$ & $\mathrm{A}$ & $\mathrm{A}$ & $\mathrm{A}$ & $\mathrm{A}$ \\
\hline D & $92 \pm$ & $247 \pm$ & $275 \pm$ & $255 \pm$ & $276 \pm$ & $267 \pm$ & $270 \pm$ & $271 \pm$ \\
& $2.0^{\mathrm{a}}$ & $3.5^{\mathrm{d}}$ & $2.5^{\mathrm{a}}$ & $2.5^{\mathrm{a}}$ & $3^{\mathrm{a}}$ & $3.5^{\mathrm{a}}$ & $3.5^{\mathrm{a}}$ & $2^{\mathrm{a}}$ \\
& $\mathrm{C}$ & $\mathrm{B}$ & $\mathrm{A}$ & $\mathrm{AB}$ & $\mathrm{A}$ & $\mathrm{AB}$ & $\mathrm{A}$ & $\mathrm{A}$ \\
\hline DI & $96 \pm$ & $250 \pm$ & $240 \pm$ & $233 \pm$ & $247 \pm$ & $244 \pm$ & $230 \pm$ & $229 \pm$ \\
& $1.50^{\mathrm{a}} \mathrm{C}$ & $5^{\mathrm{d}}$ & $5^{\mathrm{b}}$ & $1.5^{\mathrm{a}}$ & $3.5^{\mathrm{b}}$ & $2.0^{\mathrm{b}}$ & $1.5^{\mathrm{b}}$ & $4.5^{\mathrm{b}}$ \\
& & $\mathrm{A}$ & $\mathrm{AB}$ & $\mathrm{AB}$ & $\mathrm{AB}$ & $\mathrm{AB}$ & $\mathrm{AB}$ & $\mathrm{B}$ \\
\hline DR & $102 \pm$ & $263 \pm$ & $180 \pm$ & $177 \pm$ & $160 \pm$ & $150 \pm$ & $120 \pm$ & $100 \pm$ \\
& $0.5^{\mathrm{a}}$ & $1.5^{\mathrm{d}}$ & $2.5^{\mathrm{c}}$ & $0.5^{\mathrm{b}}$ & $3^{\mathrm{c}}$ & $2.5^{\mathrm{c}}$ & $0.25^{\mathrm{c}}$ & $0.5^{\mathrm{c}}$ \\
& $\mathrm{A}$ & $\mathrm{A}$ & $\mathrm{B}$ & $\mathrm{B}$ & $\mathrm{BC}$ & $\mathrm{C}$ & $\mathrm{D}$ & $\mathrm{E}$ \\
\hline DRD & $93 \pm$ & $289.0 \pm$ & $109.0 \pm$ & $100 \pm$ & $93 \pm$ & $85 \pm$ & $72 \pm$ & $66 \pm$ \\
& $3.0^{\mathrm{a}}$ & $3^{\mathrm{c}}$ & $2.5^{\mathrm{e}} \mathrm{B}$ & $0.05^{\mathrm{d}}$ & $1.5^{\mathrm{e}}$ & $1.5^{\mathrm{e}}$ & $1^{\mathrm{e}}$ & $3^{\mathrm{e}}$ \\
& $\mathrm{BC}$ & $\mathrm{A}$ & & $\mathrm{BC}$ & $\mathrm{BC}$ & $\mathrm{CD}$ & $\mathrm{D}$ & $\mathrm{D}$ \\
\hline
\end{tabular}

Each value represents mean $\pm \mathrm{SD}$

Values with non-identical superscripted $(a, b, c, d, e \&$ f) are considered as significantly different (p<0.05) among the same group of rabbits, Values with non-identical superscripted $(\mathrm{A}, \mathrm{B}, \mathrm{C}, \mathrm{D}, \mathrm{E \&} \mathrm{F})$ are considered as significantly different $(p<0.05)$ among the different groups of rabbits, $N($ number of animals $)=3$.

$\mathrm{C}=$ Control; $\mathrm{D}=$ Diabetic; $\mathrm{DI}=$ Diabetic after treated with glbcimide; $\mathrm{DR}=$ Diabetic after treated with resveratrol;DRD $=$ Diabetic after treated with its derivative

WK0=Zero week; PT=Pretreatment; WK1=First week; WK2=Second week; WK3=Third week; WK4=Fourth week; WK5=Fifth week; WK6=Sixth week.

Table (2) show significant increase in the level of Serum total protein which is a sign of renal dysfunction in the diabetic rabbits when compared to control rabbits. The diabetic rabbits treated with the resveratrol and its derivative showed significant decrease in levels of Serum Total protein $(\mathrm{p}<0.05)$. Resvertrol 
and its derivative show significant value after one week of treatment $6.6 \pm 0.03$ and $7.1 \pm 0.02$ respectively. In diabetes, protein catabolism increases due to deficiency of carbohydrate-derived energy in connection with lowserum insulin.

Table (2): The effect of resveratrol and their derivatives on serum total protein concentration $\mathrm{mg} / \mathrm{dL}$ of rabbits' groups with weeks.

\begin{tabular}{|c|c|c|c|c|c|c|c|c|}
\hline \multirow[t]{2}{*}{ Groups } & \multicolumn{8}{|c|}{ Treatment periods } \\
\hline & WKO & PT & Wk1 & Wk2 & Wk3 & Wk4 & Wk5 & Wk6 \\
\hline $\mathbf{C}$ & $\begin{array}{l}6.9 \pm \\
0.03^{a} \\
A\end{array}$ & $\begin{array}{l}7.7 \pm \\
0.07 \\
\text { A }\end{array}$ & $\begin{array}{l}7.1 \pm \\
0.02^{\mathrm{a}} \mathrm{A}\end{array}$ & $\begin{array}{l}7.1 \pm \\
0.02 \\
\mathrm{~A}\end{array}$ & $\begin{array}{l}7.1 \pm 0.0 \\
2^{\mathrm{a}} \mathrm{A}\end{array}$ & $\begin{array}{l}6.9 \pm \\
0.03 \text { a } \\
\mathrm{A}\end{array}$ & $\begin{array}{l}7 \pm \\
0.05^{\mathrm{b}} \\
\mathrm{A}\end{array}$ & $\begin{array}{l}7.1 \pm \\
0.02 \\
\text { A }\end{array}$ \\
\hline D & $\begin{array}{l}7.1 \pm \\
0.03^{a} \\
B\end{array}$ & $\begin{array}{l}5.1 \pm \\
0.05^{\mathrm{e}} \mathrm{E}\end{array}$ & $\begin{array}{l}5.2 \pm \\
0.03{ }^{\mathrm{f}} \mathrm{E}\end{array}$ & $\begin{array}{l}5 \pm 0.05 \\
\mathrm{E}\end{array}$ & $\begin{array}{l}5 \pm \\
0.05^{\mathrm{e}} \\
\mathrm{E}\end{array}$ & $\begin{array}{l}5 \pm \\
0.05^{\text {e }} \\
E\end{array}$ & $\begin{array}{l}5.3 \pm \\
0.01^{f} \\
E\end{array}$ & $\begin{array}{l}5 \\
0.05^{e^{ \pm}} \\
E\end{array}$ \\
\hline DI & $\begin{array}{l}7.0 \pm \\
0.02 \text { a } \\
\text { A }\end{array}$ & $\begin{array}{l}5.3 \pm \\
0.08^{\mathrm{e}} \mathrm{F}\end{array}$ & $\begin{array}{l}7.1 \pm \\
0.02^{\mathrm{fdc}} \\
\mathrm{A}\end{array}$ & $\begin{array}{l}7.2 \pm \\
0.06 \\
\mathrm{~A}\end{array}$ & $\begin{array}{l}7.2 \pm \\
0.06^{\mathrm{b}} \mathrm{A}\end{array}$ & $\begin{array}{l}7.1 \pm \\
0.02^{b} \\
\text { B }\end{array}$ & $\begin{array}{l}6.9 \pm \\
0.03^{a} \\
D\end{array}$ & $\begin{array}{l}7 \\
0.023^{ \pm} \\
\mathrm{A}\end{array}$ \\
\hline DR & $\begin{array}{l}7.1 \pm \\
0.02^{\mathrm{a}} \\
\text { B }\end{array}$ & $\begin{array}{l}5.3 \pm \\
0.05^{\mathrm{e}} \\
\mathrm{F}\end{array}$ & $\begin{array}{l}6.6 \pm \\
0.03 \mathrm{dc} \\
\mathrm{CB}\end{array}$ & $\begin{array}{l}6.8 \pm \\
0.10^{\mathrm{c}} \mathrm{B}\end{array}$ & $\begin{array}{l}6.8 \pm \\
0.10^{c} \mathrm{~B}\end{array}$ & $\begin{array}{l}7.2 \pm \\
0.06^{\text {ba }} \\
\text { A }\end{array}$ & $\begin{array}{l}6.8 \pm \\
0.10^{b} \\
C\end{array}$ & $\begin{array}{l}6.8 \pm \\
0.10^{\mathrm{b}} \mathrm{B}\end{array}$ \\
\hline DRD & $\begin{array}{l}7.7 \pm \\
0.07^{\mathrm{a}} \\
\mathrm{C}\end{array}$ & $\begin{array}{l}5.4 \pm \\
0.1^{\mathrm{e}} \\
\mathrm{D} \\
\end{array}$ & $\begin{array}{l}7.1 \pm \\
0.02^{\mathrm{dc}} \\
\text { A }\end{array}$ & $\begin{array}{l}7.2 \pm \\
0.06^{\mathrm{b}} \\
\text { B }\end{array}$ & $\begin{array}{l}7.2 \pm \\
0.06^{\mathrm{b}} \mathrm{B}\end{array}$ & $\begin{array}{l}7.2 \pm \\
0.06 \\
B\end{array}$ & $\begin{array}{l}7.4 \pm \\
0.02^{a} \\
\text { A }\end{array}$ & $\begin{array}{l}7.3 \pm \\
0.02^{\mathrm{a}} \mathrm{B}\end{array}$ \\
\hline
\end{tabular}

Each value represent mean $+\mathrm{SD}$

Values with non-identical superscripted $(a, b, c, d, e \&$ f) are considered as significantly different $(\mathbf{p}<0.05)$ among the same group of rabbits, Values with non-identical superscripted $(\mathrm{A}, \mathrm{B}, \mathrm{C}, \mathrm{D}, \mathrm{E \&} \mathrm{F})$ are considered as significantly different $(p<0.05)$ among the different groups of rabbits, $N($ number of animals $)=3$.

$\mathrm{C}=$ Control ;D=Diabetic; I=Diabetic after treated with glbcimide;DR= Diabetic after treated with resveratrol;DRD= Diabetic after treated with derivative

The results showed significant increase in the level of serum albumin which is asign of renal dysfunction in the diabetic rabbits when compared with control rabbits (Table 3). The diabetic rabbits treated with resveratrol and their derivatives decrease levels of Serum Albumin significantly $(\mathrm{p}<0.05)$. The results showed significant decrease in the level of serum urea of rabbits treated with resveratrol and its derivative $(\mathrm{p}<0.05)$. Resveratrol and its derivative show significant value after one week of treatment $3.7 \pm 0.1$ and $4.2 \pm 0.05$, respectively. 
Antidiabetic and antihyperglycemica effects of resvertrol and its derivative.....

Table (3): The effect of resveratrol and its derivative on serum albumin concentration (mg/dL) of Rabbits' groups with weeks.

\begin{tabular}{|c|c|c|c|c|c|c|c|c|}
\hline \multirow[t]{2}{*}{ Groups } & \multicolumn{8}{|c|}{ Treatment periods } \\
\hline & WKO & PT & Wk1 & Wk2 & Wk3 & Wk4 & Wk5 & Wk6 \\
\hline $\mathbf{C}$ & $\begin{array}{l}4.4 \pm \\
0.1^{\mathrm{a}} \\
\mathrm{A} \\
\end{array}$ & $\begin{array}{l}4.3 \pm \\
0.07^{\mathrm{a}} \\
\mathrm{A}\end{array}$ & $\begin{array}{l}4.3 \pm \\
0.07^{\mathrm{a}} \\
\mathrm{A}\end{array}$ & $\begin{array}{l}4.2 \pm \\
0.05^{\mathrm{a}} \\
\mathrm{A}\end{array}$ & $\begin{array}{l}4.4 \pm \\
0.10^{\mathrm{a}} \\
\mathrm{A}\end{array}$ & $\begin{array}{l}4.2 \pm \\
0.05^{\mathrm{a}} \\
\mathrm{A}\end{array}$ & $\begin{array}{l}4.2 \pm \\
0.05^{\mathrm{a}} \\
\mathrm{A}\end{array}$ & $\begin{array}{l}4.3 \pm \\
0.08^{a} \\
\text { A }\end{array}$ \\
\hline D & $\begin{array}{l}4.2 \pm \\
0.1^{\mathrm{a}} \\
\mathrm{A}\end{array}$ & $\begin{array}{l}2.9 \pm \\
0.15^{b} \\
\text { B }\end{array}$ & $\begin{array}{l}2.9 \pm \\
0.22^{\mathrm{b}} \\
\text { B }\end{array}$ & $\begin{array}{l}2.6 \pm \\
0.15^{\mathrm{b}} \\
\text { B }\end{array}$ & $\begin{array}{l}2.7 \pm \\
0.17^{b} \\
\text { B }\end{array}$ & $\begin{array}{l}2.9 \pm \\
0.22^{\mathrm{b}} \\
\text { B }\end{array}$ & $\begin{array}{l}2.9 \pm \\
0.1 \\
\text { B }\end{array}$ & $\begin{array}{l}2.7 \pm \\
0.18^{b} \\
\text { B }\end{array}$ \\
\hline DI & $\begin{array}{ll}4.3 & \pm \\
0.09^{\mathrm{a}} \mathrm{A}\end{array}$ & $\begin{array}{l}3.0 \pm \\
0.15^{\mathrm{b}} \mathrm{B}\end{array}$ & $\begin{array}{l}3.9 \pm \\
0.23^{\mathrm{a}} \\
\text { A }\end{array}$ & $\begin{array}{l}4.5 \pm \\
0.25^{\mathrm{a}} \\
\text { A }\end{array}$ & $\begin{array}{l}4.4 \pm \\
0.20^{\mathrm{a}} \\
\text { A }\end{array}$ & $\begin{array}{l}4.2 \pm \\
0.04^{\mathrm{a}} \\
\mathrm{A}\end{array}$ & $\begin{array}{l}4.4 \pm \\
0.1^{\mathrm{a}} \\
\mathrm{A}\end{array}$ & $\begin{array}{l}4.1 \\
\pm 0.02^{\mathrm{a}} \\
\text { A }\end{array}$ \\
\hline DR & $\begin{array}{l}4.2 \pm \\
0.05^{\mathrm{a}} \mathrm{A}\end{array}$ & $\begin{array}{l}3.1 \pm \\
0.03^{\mathrm{b}} \mathrm{B}\end{array}$ & $\begin{array}{l}3.7 \pm \\
0.1^{\mathrm{a}} \\
\text { A }\end{array}$ & $\begin{array}{l}3.8 \pm \\
0.20^{\mathrm{a}} \\
\mathrm{A}\end{array}$ & $\begin{array}{l}4.0 \pm \\
0.10^{\mathrm{a}} \\
\text { A }\end{array}$ & $\begin{array}{l}4.4 \pm \\
0.2^{a} \\
\text { A }\end{array}$ & $\begin{array}{l}4.4 \pm \\
0.1^{\mathrm{a}} \\
\mathrm{A}\end{array}$ & $\begin{array}{l}4.2 \pm \\
0.05^{\mathrm{a}} \mathrm{A}\end{array}$ \\
\hline DRD & $\begin{array}{l}4.2 \pm \\
0.06^{\mathrm{a}} \mathrm{A}\end{array}$ & $\begin{array}{l}2.9 \pm \\
0.21^{\mathrm{b}} \mathrm{B}\end{array}$ & $\begin{array}{l}4.2 \pm \\
0.05^{\mathrm{a}} \\
\mathrm{A}\end{array}$ & $\begin{array}{c}4.3 \pm \\
0.08^{\mathrm{a}} \\
\mathrm{A}\end{array}$ & $\begin{array}{l}4.3 \pm \\
0.07^{\mathrm{a}} \\
\mathrm{A}\end{array}$ & $\begin{array}{l}4.4 \pm \\
0.02^{\mathrm{a}} \\
\text { A }\end{array}$ & $\begin{array}{l}4.4 \pm \\
0.06 \\
\mathrm{~A}\end{array}$ & $\begin{array}{l}4.3 \pm \\
0.10^{\mathrm{a}} \mathrm{A}\end{array}$ \\
\hline
\end{tabular}

Each value represent mean $\pm \mathrm{SD}$

Values with non-identical superscripted $(a, b, c, d, e \&$ f) are considered as significantly different $(\mathrm{p}<0.05)$ among the same group of rabbits, Values with non-identical superscripted $(\mathrm{A}, \mathrm{B}, \mathrm{C}, \mathrm{D}, \mathrm{E \&} \mathrm{F})$ are considered as significantly different $(p<0.05)$ among the different groups of rabbits, $N($ number of animals) $=3$.

$\mathrm{C}=$ Control ;D=Diabetic; $\mathrm{I}=$ Diabetic after treated with glbcimide;DR= Diabetic after treated with resveratrol $; \mathrm{DRD}=$ Diabetic after treated with derivative.

WK0=Zero week; PT=Pretreatment; WK1=First week; WK2=Second week; WK3=Third week; WK4=Fourth week; WK5=Fifth week; WK6=Sixth week.

The clinical significance of the creatinine level in plasma or serum is usually determined in conjugation with the plasma urea level since there is an increase in both levels in postrenal azotemia, while the creatinine clearance (CC), or urine levels, are diminished. The diabetic rabbits treated with the resveratrol and its derivative showed significant decreased levels of serum creatinine $(p<0.05)$. This further shows the ability of the resveratrol and its derivative in treating diabetes associated renal complications and also supports the usage of these compounds for kidney diseases. Resveratrol derivative show significant value after 2 weeks of treatment $1.1 \mathrm{mg} / \mathrm{dL} \pm 0.03$. and resveratrol failed to reach to normal value as shown in table (4). Serum urea and creatinine levels increased significantly in the diabetic control group with respect to the control group. The levels of these parameters restored towards the control level after administration of resveratrol and its derivative to the diabetic rabbits. This has been indicated here by high levels of serum urea and creatinine. High-serum creatinine level is also the sign of muscle wastage. 
Antidiabetic and antihyperglycemica effects of resvertrol and its derivative.....

Table (4): The effect of resveratrol and their derivatives on serum creatinine concentration $\mathrm{mg} / \mathrm{dL}$ of rabbits' groups with weeks.

\begin{tabular}{|c|c|c|c|c|c|c|c|c|}
\hline \multirow[t]{2}{*}{ Groups } & \multicolumn{8}{|c|}{ Treatment periods } \\
\hline & WK0 & PT & Wk1 & Wk2 & Wk3 & Wk4 & Wk5 & Wk6 \\
\hline C & $\begin{array}{c}1 \pm \\
0.02^{\mathrm{a}} \\
\mathrm{A}\end{array}$ & $\begin{array}{c}0.9 \pm \\
0.024^{\mathrm{c}} \\
\mathrm{A}\end{array}$ & $\begin{array}{c}1 \pm \\
0.02^{\mathrm{d}} \\
\text { A }\end{array}$ & $\begin{array}{c}1 \pm \\
0.02^{\mathrm{f}} \\
\text { A }\end{array}$ & $\begin{array}{c}0.9 \pm \\
0.02^{\mathrm{f}} \\
\text { A }\end{array}$ & $\begin{array}{c}0.9 \pm \\
0.024^{f} \\
\text { A }\end{array}$ & $\begin{array}{c}1.1 \pm \\
0.023^{\mathrm{d}} \\
\mathrm{A}\end{array}$ & $\begin{array}{c}0.9 \pm \\
0.24^{\mathrm{a}} \\
\text { A }\end{array}$ \\
\hline $\mathrm{D}$ & $\begin{array}{c}1.1 \pm \\
0.02^{\mathrm{a}} \mathrm{C}\end{array}$ & $\begin{array}{l}3.2 \pm \\
0.15^{\mathrm{a}}\end{array}$ & $\begin{array}{c}3.6 \pm \\
0.16^{\mathrm{a}} \\
\text { A }\end{array}$ & $\begin{array}{c}3.6 \pm \\
0.16^{\mathrm{a}} \\
\text { A }\end{array}$ & $\begin{array}{c}3.9 \pm \\
0.2^{\mathrm{a}} \\
\text { A }\end{array}$ & $\begin{array}{c}3.9 \pm \\
0.2^{\mathrm{a}} \\
\mathrm{A}\end{array}$ & $\begin{array}{c}3.8 \pm \\
0.35^{\mathrm{a}} \\
\mathrm{A}\end{array}$ & $\begin{array}{c}3.9 \pm \\
0.2^{\mathrm{a}} \\
\mathrm{A}\end{array}$ \\
\hline DI & $\begin{array}{c}0.9 \pm \\
0.24^{\mathrm{a}} \mathrm{D}\end{array}$ & $\begin{array}{c}2.8 \pm \\
0.12^{\mathrm{b}} \\
\mathrm{B}\end{array}$ & $\begin{array}{c}3.2 \pm \\
0.15^{\mathrm{b}} \\
\text { A }\end{array}$ & $\begin{array}{c}3.0 \pm \\
0.14^{\mathrm{b}} \\
\mathrm{A}\end{array}$ & $\begin{array}{c}3.1 \pm \\
0.15^{\mathrm{b}} \\
\text { A }\end{array}$ & $\begin{array}{c}2.7 \pm \\
0.14^{\mathrm{a}} \\
\mathrm{B}\end{array}$ & $\begin{array}{c}2.2 \pm \\
0.17^{\mathrm{b}} \\
\mathrm{C}\end{array}$ & $\begin{array}{c}2.3 \pm \\
0.11^{\mathrm{b}} \mathrm{C}\end{array}$ \\
\hline DR & $\begin{array}{c}1.1 \pm \\
0.023^{\mathrm{a}} \\
\mathrm{C}\end{array}$ & $\begin{array}{c}3.0 \pm \\
0.14^{\mathrm{ab}} \\
\mathrm{A}\end{array}$ & $\begin{array}{c}3.0 \pm \\
0.14^{\mathrm{b}} \\
\mathrm{A}\end{array}$ & $\begin{array}{c}2.9 \pm \\
0.10^{\mathrm{b}} \\
\mathrm{B}\end{array}$ & $\begin{array}{c}2.8 \pm \\
0.12^{\mathrm{c}} \\
\mathrm{BC}\end{array}$ & $\begin{array}{c}2.3 \pm \\
0.15^{\mathrm{b}} \\
\mathrm{C}\end{array}$ & $\begin{array}{c}2.3 \pm \\
0.15^{\mathrm{b}} \\
\mathrm{D}\end{array}$ & $\begin{array}{c}2.2 \pm \\
0.1^{\mathrm{b}} \\
\mathrm{D}\end{array}$ \\
\hline DRD & $\begin{array}{c}1.0 \pm \\
0.02^{\mathrm{a}} \\
\mathrm{B}\end{array}$ & $\begin{array}{c}3.3 \pm \\
0.24^{\mathrm{a}} \\
\text { A }\end{array}$ & $\begin{array}{c}1.2 \pm \\
0.02^{\mathrm{d}} \\
\text { B }\end{array}$ & $\begin{array}{c}1.1 \pm \\
0.03^{\text {ef }} \\
B\end{array}$ & $\begin{array}{c}1.1 \pm \\
0.03^{\mathrm{f}} \\
B\end{array}$ & $\begin{array}{c}1.0 \pm \\
0.05^{\mathrm{f}} \\
B\end{array}$ & $\begin{array}{c}1.0 \pm \\
0.03^{\mathrm{d}} \\
B\end{array}$ & $\begin{array}{c}1.0 \pm \\
0.02^{\mathrm{d}} \\
\mathrm{B}\end{array}$ \\
\hline
\end{tabular}

Each value represent mean $+\mathrm{SD}$

Values with non-identical superscripted $(\mathbf{a}, \mathrm{b}, \mathrm{c}, \mathrm{d}, \mathrm{e} \& \mathrm{f})$ are considered as significantly different $(p<0.05)$ among the same group of rabbits, Values with non-identical superscripted $(\mathrm{A}, \mathrm{B}, \mathrm{C}, \mathrm{D}, \mathrm{E \&} \mathrm{F})$ are considered as significantly different $(p<0.05)$ among the different groups of rabbits.

$\mathrm{C}=$ Control ; $\mathrm{D}=$ Diabetic; $\mathrm{I}=$ Diabetic after treated with glbcimide;DR= Diabetic after treated with resveratrol;DRD= Diabetic after treated with derivative.

The results showed significant increase in the level of serum insulin of rabbits treated with resveratrol and its derivative significantly $(\mathrm{p}<0.05)$. Derivative show significant value after one week of treatment 0.8 $\square$ mole/ $\mathrm{L} \pm 0.02$ respectively shown in table (5).

Many herbal plants' extracts was able to recover the protein metabolic disorders possibly by stimulating the existing $\beta$ cells and or by regenerating $\beta$ cells like other plant products. Since insulin inhibits adipose tissue hormone sensitive lipase and reduces lipolysis, the aqueous-methanolic extract of $S$ mahagoni seed may correct the above mentioned disorders by mimicking insulin action [14].

Table (5): The effect of resveratrol and its derivative on serum Insulin concentration ( $\mu \mathrm{mole} / \mathrm{L})$ of rabbits' groups with weeks.

\begin{tabular}{|c|c|c|c|c|c|c|c|c|}
\hline \multirow[t]{2}{*}{ Groups } & \multicolumn{8}{|c|}{ Treatment periods } \\
\hline & WKO & PT & Wk1 & Wk2 & Wk3 & Wk4 & Wk5 & Wk6 \\
\hline $\mathbf{C}$ & $\begin{array}{l}0.8 \pm 0.02^{\mathrm{a}} \\
\mathrm{A}\end{array}$ & $\begin{array}{l}0.9 \pm \\
0.024^{\mathrm{c}} \\
\text { B }\end{array}$ & $\begin{array}{l}1 \pm 2.02^{\mathrm{d}} \\
\mathrm{C}\end{array}$ & $\begin{array}{l}1 \pm 0.02^{f} \\
C\end{array}$ & $\begin{array}{l}0.9 \pm \\
0.02^{\mathrm{f}} \mathrm{B}\end{array}$ & $\begin{array}{l}0.9 \pm \\
0.024^{\mathrm{f}} \mathrm{B}\end{array}$ & $\begin{array}{l}1.1 \pm \\
0.023^{\mathrm{d}} \\
C\end{array}$ & $\begin{array}{l}0.9 \pm \\
0.24^{\mathrm{a}} B\end{array}$ \\
\hline D & $\begin{array}{l}1.1 \pm 0.02^{\mathrm{a}} \\
\mathrm{B}\end{array}$ & $\begin{array}{l}0.02 \pm 0 . \\
15^{\mathrm{e}} \\
\text { A }\end{array}$ & $\begin{array}{l}0.03 \pm \\
0.16^{\mathrm{e}} \\
\mathrm{A}\end{array}$ & $\begin{array}{l}0.03 \pm \\
0.16^{\mathrm{e}} \mathrm{A}\end{array}$ & $\begin{array}{l}0.03 \pm \\
0.2^{\mathrm{e}} \\
\mathrm{A}\end{array}$ & $\begin{array}{l}0.03 \pm \\
0.2^{\mathrm{e}^{\mathrm{a}}} \\
\mathrm{A}\end{array}$ & $\begin{array}{l}0.03 \pm \\
0.35^{\mathrm{e}} \\
\mathrm{A}\end{array}$ & $\begin{array}{ll}0.03 & \pm \\
0.2^{\mathrm{e}} & \\
\mathrm{A} & \end{array}$ \\
\hline DI & $\begin{array}{l}0.9 \pm \\
0.24^{\mathrm{a}} \\
\mathrm{A}\end{array}$ & $\begin{array}{l}0.02 \pm 0 . \\
12^{\mathrm{e}} \\
\mathrm{A}\end{array}$ & $\begin{array}{l}0.3 \pm \\
0.15^{\mathrm{e}} \\
\mathrm{A}\end{array}$ & $\begin{array}{l}0.3 \pm \\
0.14^{\mathrm{e}} \mathrm{A}\end{array}$ & $\begin{array}{l}0.3 \pm \\
0.15^{\mathrm{d}} \\
\mathrm{A}\end{array}$ & $\begin{array}{l}0.27 \pm \\
0.14^{d} \\
\mathrm{~A}\end{array}$ & $\begin{array}{l}0.22 \pm \\
0.17^{\mathrm{d}} \\
\mathrm{A}\end{array}$ & $\begin{array}{l}0.23 \pm 0.1 \\
1^{\mathrm{d}} \mathrm{A}\end{array}$ \\
\hline DR & $\begin{array}{l}1.1 \pm \\
0.023^{\mathrm{a}} \mathrm{BA}\end{array}$ & $\begin{array}{l}0.02 \pm 0 . \\
14^{\mathrm{e}} \mathrm{A}\end{array}$ & $\begin{array}{l}0.3 \pm \\
0.14^{\mathrm{e}} \\
\mathrm{A} \\
\end{array}$ & $\begin{array}{l}0.32 \pm \\
0.10^{\mathrm{d}} \mathrm{A}\end{array}$ & $\begin{array}{l}0.38 \pm \\
0.12^{\mathrm{d}} \\
\mathrm{A}\end{array}$ & $\begin{array}{l}0.38 \pm \\
0.15^{\mathrm{d}} \\
\mathrm{A}\end{array}$ & $\begin{array}{l}0.4 \pm \\
0.15^{\mathrm{d}} \\
\text { A }\end{array}$ & $\begin{array}{ll}0.42 & \pm \\
0.1^{\mathrm{c}} & \\
\mathrm{A} & \\
\end{array}$ \\
\hline DRD & $\begin{array}{ll}1.0 & \pm \\
0.02^{\mathrm{a}} & \\
\mathrm{B} & \end{array}$ & $\begin{array}{l}0.03 \pm \\
0.24^{\mathrm{e}} \mathrm{A}\end{array}$ & $\begin{array}{l}0.8 \pm \\
0.02^{\text {cd }} \\
\mathrm{A}\end{array}$ & $\begin{array}{l}0.9 \pm \\
0.03^{\mathrm{bc}} \mathrm{A}\end{array}$ & $\begin{array}{l}1 \pm \\
0.03^{\text {ab }} \\
\mathrm{A}\end{array}$ & $\begin{array}{l}1.2 \pm \\
0.05^{\mathrm{a}} \\
\mathrm{A}\end{array}$ & $\begin{array}{l}1.3 \pm \\
0.03^{\mathrm{a}} \\
\mathrm{A}\end{array}$ & $\begin{array}{l}1.35 \pm 0.0 \\
2^{\mathrm{a}} \mathrm{A}\end{array}$ \\
\hline
\end{tabular}


Each value represent mean $+\mathrm{SD}$

Values with non-identical superscripted $(a, b, c, d, e \&$ f) are considered as significantly different $(\mathrm{p}<0.05)$ among the same group of rabbits, Values with non-identical superscripted $(\mathrm{A}, \mathrm{B}, \mathrm{C}, \mathrm{D}, \mathrm{E \&} \mathrm{F})$ are considered as significantly different $(p<0.05)$ among the different groups of rabbits, $N($ number of animals) $=3$.

$\mathrm{C}=$ Control ;D=Diabetic; $\mathrm{I}=$ Diabetic after treated with glbcimide;DR= Diabetic after treated with resveratrol;DRD= Diabetic after treated with derivative. WK0=Zero week; PT=Pretreatment; WK1=First week; WK2=Second week; WK3=Third week; WK4=Fourth week; WK5=Fifth week; WK6=Sixth week.

\subsection{Examinations of the Liver and Pancreas Sections}

These rabbits were excluded from the study. Necropsy was performed after euthanasia, or death in one rabbit, in the individual group and in one normal rabbit. The light microscopic examination by specific staining of pancreatic beta cells in control tissues showed normal appearance of islet of langerhans which was not observed in diabetic pancreas figure(1) and (2) respectively. The pancreas was examined mainly, especially the pancreatic islets. Pancreatic section of rabbits treated with resveratrol and its derivative showed no notable histological changes. Secretion from remnant pancreatic beta cells which in turn enhances the glucose utilization by peripheral tissue of diabetic rabbits. The histopathology examination showed that pancreatic islets disappeared and that the exocrine tissue remained relatively inaffected; any remaining islets were considerably atrophied compared with the normal pancreas. In the pancreatic section of rabbits treated with derivative no notable histological changes appear figures (3) and (4).

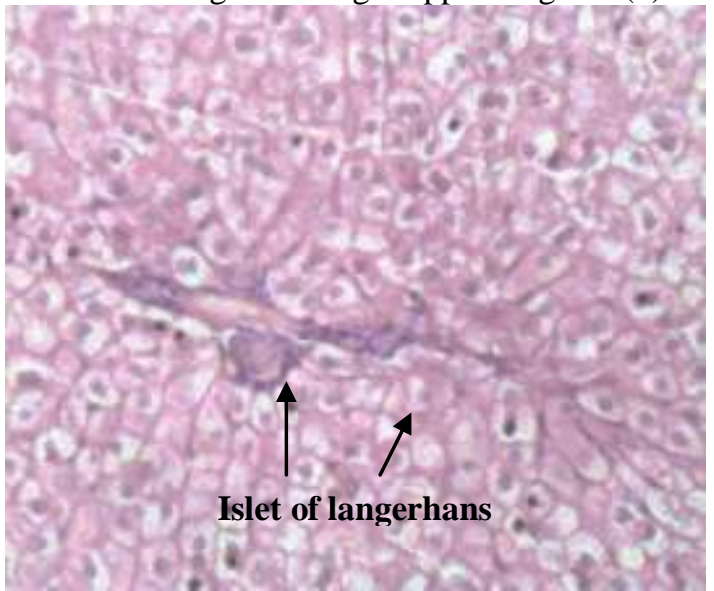

Figure (1) Section of control non-diabetic pancreas Rabbit with normal looking appearance of exocrine of Islet of langerhans (X200) (H\&E).

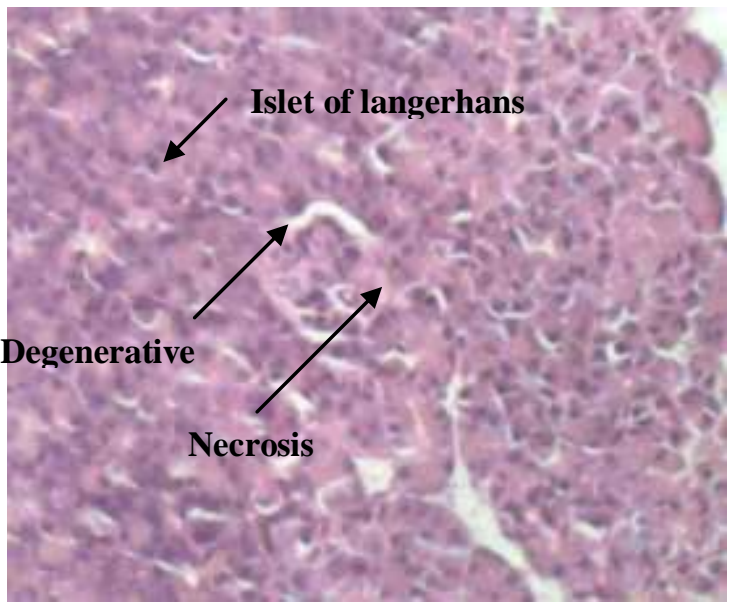

Figure: (3) Section of diabetic pancreas rabbit

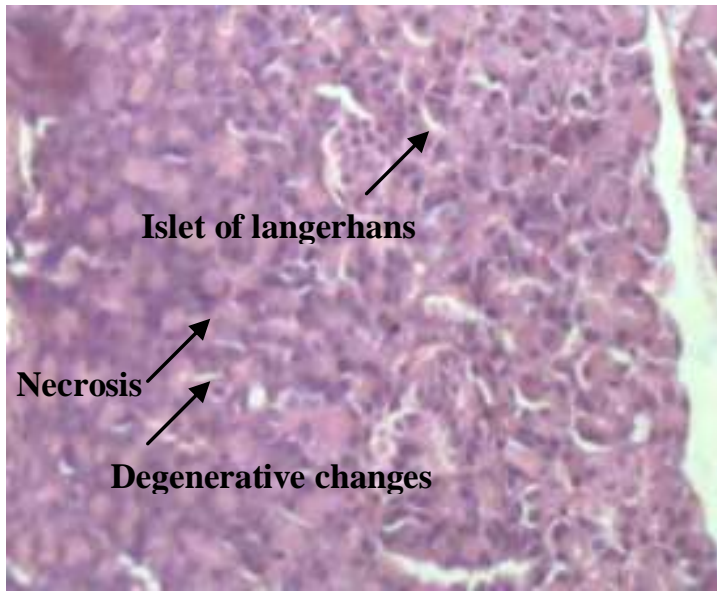

Figure (2) Section of diabetic pancreas rabbit shows degenerative changes \& necrosis \& endocrine (Islet of langerhans) (X200) (H\&E).

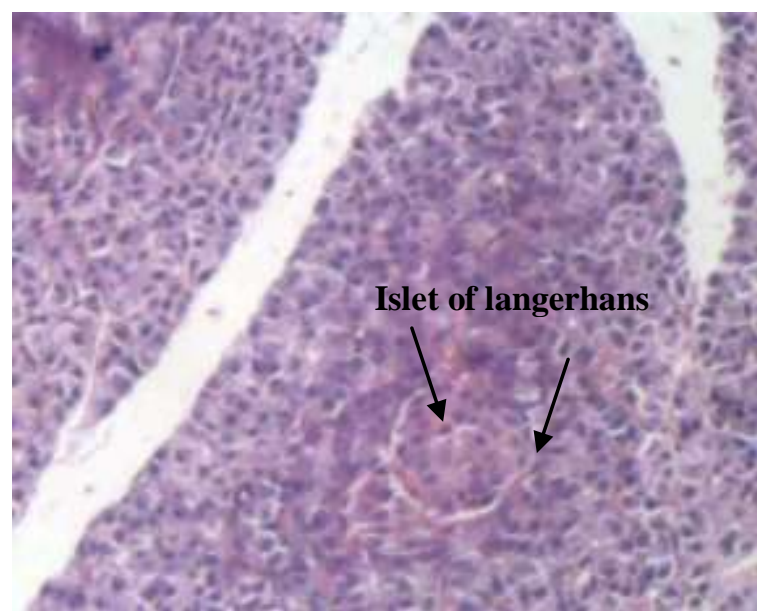

Figure :(4) Section of diabetic pancreas rabbit 
Antidiabetic and antihyperglycemica effects of resvertrol and its derivative.....

after treated with resveratriol shows degenerative necrosis of islet of langerhans (X200) (H\&E). after treated with derivative shows normal \& looking of islet of langerhans (X200) (H\&E)

\section{DISCUSSION}

Thus, the significant anti-diabetic effect of resveratrol and its derivative may be due to the presence of phenolics and or their synergistic properties. This may be due to the presence of hydroxyl as shown in resveratrol and heterocyclic amide rings as shown in its derivative [15]. These results agreed with the study of diabetes induced by Streptozotocin resulted in a significant elevation in blood glucose level in comparison with the control group after administration of aqueous methanolic (2:3) extract of $S$. mahagoni seed to the diabetic rabbits for 21 days [14]. A significant reduction in blood glucose level was noted this was close to the control level.

The results also agreed with the study of the protective effect of plant compound idopyranose isolated from the plant, Vitex negundo evaluated against streptozotocin-induced diabetics. Wister rats showed significant reduction in blood glucose [16].

These findings indirectly show that the blood glucose levels in the groups treated with resveratrol and its derivative may have been lowered not by insulin but by derivatives; this is because almost all of the pancreatic islet beta cells were destroyed and therefore resulted in insulin deficiency [17].

The anti- hyperglycemic activity of resveratrol and its derivative probably caused by stimulation of insulin have also been reported to have a similar effect as observed in the present investigation. Significant increase in the lymphocytes induced by resveratrol and its derivative reflects possible imunomodulatory effects alloxan-induced diabetic rats. It is well known that certain polyphenols and their derivatives exhibit hypoglycemic activity and are known for their ability of beta-cell regeneration of pancreas. It can therefore be concluded that the preliminary study shows that the resveratrol and its derivative decreases the blood glucose level in diabetic rabbits.

Correction of oxidative injury which is associated with diabetes is another possibility of the recovery in glycemic disorders. Resveratrol and their derivatives are able to recover the protein metabolic disorders possibly by stimulating the existing $\beta$-cells and or by regenerating $\beta$ cells like other plant products [18].

\section{CONCLUSION:}

The results suggested the resveratrol and its derivative are safe and potent hypoglycemic agent which is capable for normalizing other biochemical and hematological abnormalities associated with diabetes mellitus type 2 .

\section{REFERENCES}

[1]. F.A.Tomas- Barberan and R.J.Robins. Phytochemistry of fruit and vegetables. (Clarendon press- Oxford. New York. 1997).

[2]. J.B. Harborne. Phytochemical Methods.( Chapman and Hall. London. 1984).

[3]. J.Bruneton, Pharmacognosy phytochemistry Medicinal plants , ( $2^{\text {nd }}$ edition , TEC 8 DOC .Paris, France) 1999.

[4]. L.Barclay, . Growing evidence links, resveratrol to extended life span. Life Extension Magazine , 13, 2007, 1-8.

[5]. Y.D.Kim, K.G.Park, Y.S.Lee, Metformin inhibits hepatic gluconeogenesis through AMP-activated protein kinase-dependent regulation of the orphan nuclear receptor SHP. Diabetes. 57(,2) : 2008, 306-314.

[6]. T.Pussa T, J.Floren, P.Huldhepp and A. Raal. Survery of grape wine vitis vinefera sternpoly phenols by liquid chroma tography - Diode array detection tandem mass, spectrometry. J. Agric. Food chem, (54): 2006. 7488-7494

[7]. T.M.Bass, D.Weinkove, K.Houthoofd, D.Gems, L. Partridge, Effects of resveratrol on lifespan in Drosophila melanogaster and Caenorhabditis elegans. Mechanisms of Ageing and Development .(128) .10: 2007, 546-52.

[8]. B.D.Gehm, J.M.McAndrews, P.Y.Chien, J.L.Jameson, Resveratrol, a polyphenolic compound found in grapes and wine, is an agonist for the estrogen receptor. Proceedings of the National Academy of Sciences of the United States of America 94, (25) $1997: 14138-43$

[9]. W.W. Stephen ; N.H.J. Kelly. Organic Chemistry. 2006, 71, 1080

[10]. D.S. Young, et al; Effect of drugs on clinical laboratory tests, $5^{\text {th }}$ ed, AACC Press,(2000).

[11]. L.C. Junqueira, and J.Carneiro Basic Histology, tenth edition. The McGraw-Hill Companies, USA.(2003).

[12]. D.Duncan , (1955). Multiple range and Multiple F-test Biometrics -11:1-42.

[13]. R.G.Steel, and J.H.Torrie ,(1980) Principles and procedures of statistics., $2^{\text {nd }}$.ed,McGraw.Hill Book Co .New York, Ny.

[14]. I. Debasis De, K. Chatterjee, A. K. Ali, B. Tushar Kanti and G.Debidas, Antidiabetic Potentiality of the Aqueous-Methanolic Extract of Seed of Swieteniamahagoni (L.) Jacq. in Streptozotocin-Induce DiabeticMale Albino Rat: A Correlative and Evidence-Based Approach with Antioxidative and Antihyperlipidemic Activities. Hindawi Publishing Corporation EvidenceBased Complementary and Alternative Medicine, Article ID 892807, 2011, 11.

[15]. J.H.Black and J.M.Beale ,Wilson and Gisvold's Textbook of Organic Medicinal and Pharmaceutical Chemistry. (Lippincott Willamsand Wilkins. London. 2004). 
[16]. R. Manikandan, R. Sundaram, P. Srinivasan, S. Beulaja and C. Arulvasu. Isolation of 1, 2 di-substituted idopyranose from Vitex negundo and its effects on diabetic ratsInternational J. of Pharmaceuticals Analysis, 1, 2, 2009, pp-4-10.

[17]. C.M.Cam, H.G.Cros,J.J.Serrano,R.Lazaro,H.J.McNeill.In vivo antidiabetic actions of naglivan,an organic vanadyl compound instreptozotocin-induced diabetes. Diabetes Res. and Clin. Practice,(20), 2003, 111-121.

[18]. M. Kaleem, P. Medha, Q.U. Ahmed, M. Asif, B. Bano, Beneficial effects of Annona squamosa extract in streptozotocin-induced diabetic rats Singapore Med J.49,(10), $2008: 800$ 